\title{
An allometric model for trees
}

\author{
C. Fleurant ${ }^{\mathrm{a}, *}$ J. Duchesne ${ }^{\mathrm{a}}$ P. Raimbault ${ }^{\mathrm{b}}$ \\ ${ }^{a}$ Landscape Laboratory \\ ${ }^{\mathrm{b}}$ Horticultural Department
}

National Institute of Horticulture, 2 rue Le Nôtre, 49045 Angers cedex 01

France

\begin{abstract}
This paper presents a general mathematic model for a morphometric description of trees. This model is based on the introduction of the fractal theory, and more particularly of the concept of self-similarity, into a statistical physics rationale. Fractal theory provides the necessary tools to describe the complexity of tree structure. Statistics, when applied to physics, makes it possible to explain the properties of complex objects starting from their components. The combination of both tools allowed us to develop a theoretical model which is anything other than the probability density function of the morphometric lengths of trees. An example of validation of this law is given here: the theoretical model is compared with the morphometric lengths of Cupressocyparis.
\end{abstract}

Key words: fractal, topology, tree, statistical physics, branching networks.

\footnotetext{
$\bar{*}$ Corresponding author.

Email address: cyril.fleurant@inh.fr (C. Fleurant).
} 


\section{Introduction}

Figure 1, shows two branching networks of a completely different nature. One is a tree and the other is a stream network. Both networks are threedimensional but Figure 1 only represents their projected shape onto a plane. The striking similarity between their structures led us to describe them using this analogy.

\subsection{Stream networks}

Well before Mandelbrot (1988), developed his fractal theory, branching networks and more particularly stream networks structure had been studied very accurately. In fact, geological scientists and hydrologists such as Horton (1945), Schumm (1956), Strahler (1957) or Shreve (1969) took an interest in analysing the complex ordering of these networks. They made topological and morphometric analyses (Kirshen \& Bras (1983)) which can be applied to all branching networks that are three-dimensional and organised into a hierachy. A stream network is made up of several headwaters which represent its origins upstream, and an outlet which is a unique point downstream. The headwaters merge to form confluences, thus creating stream stretches that merge in their turn, and so forth down to the outlet. A stream stretch is a segment of a stream network situated between two confluences. The precise position of a stream segment within the whole river network can be identified by classifying the network. Many classification systems have been put forward (Horton (1945)) but we have decided upon Strahler's system (1952) which is the most widely used. The classification system (see diagram on Figure 2) is as follows: 
- headwaters are first order stream segments;

- when two stream segments within the same order $i$ merge, the stream segment resulting from this confluence is within order $i+1$;

- when two stream segments within different orders, $i$ and $j$ merge, the stream segment resulting from this confluence is within order $\max (i, j)$.

Strahler's classification thus makes it possible to organise the different segments of a stream network into a hierarchy. Consequently, the stream outlet will have the highest index value, corresponding to the river network order. Starting from the segments and their classification, it is possible to identify one segment as being representative of all segments within the same order.

Hydrologists relied on this classification to put forward general geometric laws concerning the ordering of stream networks. Among them, Horton's laws (1945), describe the way stream networks are organised. These laws express the so-called bifurcation ratio $R_{B}$ and length ratio $R_{L}$, also known as Horton's ratios. A great number of experimental studies on stream networks (Barbera \& Rosso (1987), Feder (1988), Barbera \& Rosso (1989), Tarboton et al. (1990), Barbera \& Rosso (1990), Rosso et al. (1991)) reveal that these ratios are rather stable and fluctuate between 3 and 5 for $R_{B}$ and between 1.5 and 3.5 for $R_{L}$. Nevertheless, it seems that Horton's laws are governed by the homogeneity of several factors, such as geological factors (Abrahams (1984)).

As Smart (1968) and Dooge (1986) point out: "these laws have a statistical nature". It means that one should not expect an accurate description of a stream network in isolation but, that these laws indicate an average pattern, a general trend for a large number of networks. 
Besides Horton's laws, Tokunaga's laws (1984), should also be mentioned. They are more general and give a more accurate description of the structure of stream networks. In fact, Tokunaga branching ratio indicates the average number of $i$ order segments that merge with $j$ order stream segments $(i<$ j). Actually, this taxonomy is similar to Horton's laws, especially when the drainage density of the stream network is equal to the stream length in its basin area (Dodds \& Rothman (2000)).

Fractal geometry, which has been developed by Mandelbrot (1988), makes it possible to describe complex objects having an irregular structure whereas usual Euclidian geometry does not.

In many scientific fields, fractal theory has imposed itself very quickly as an essential means to describe natural objects. References to numerous works show the multidisciplinary use of this theory (Mandelbrot (1988), Mandelbrot (1995), Barnsley (1988), Feder (1988), Schroeder (1991), Peitgen H. \& Saupe (1992)) as well as its applications in earth sciences (Korvin (1992), Turcotte (1992), Vicsek (1994), Barton (1995), Rodriguez-Iturbe \& Rinaldo (1997)), landscape sciences (Frankhauser (1994), Dauphiné (1995), Roland \& Fleurant (2002)), engineering sciences (Nakagawa (1999), Dekking (1999)), physical sciences (Gouyet (ales), Sapoval (1997)), and life sciences (West et al. (1997), Douady \& Couder (1992)). The fractal structure of stream networks has been studied more specifically by (Tarboton et al. (1988), Barbera \& Rosso (1987), Barbera \& Rosso (1989), Nikora (1991), Nikora et al. (1993), Nikora \& Sapozhnikov (1993)).

The above-mentioned authors have studied the self-similarity of the structure of stream networks and its relationship with the fractal dimension. They also demonstrated that self-similarity includes a statistical dimension for these 
stream networks that are in fact natural fractals, and relies heavily on the power laws; in fact the latter give evidence of similar characteristics at different scales. The Table 1 gives a synthesis of the various expressions of fractal dimension which have been found out by the above-mentioned authors. It can be noticed that these expressions take Horton's ratios into account and assume that stream networks are self-similar, whereas many studies reveal that they are self-affine Nikora et al. (1993) Even though the expression put forward by Nikora et al (1993) is not so wrong, the most accurate method to determine the fractal dimension of stream networks seems to be the box counting method or mass method.

Beyond these considerations, it should be added that calculation of the fractal dimension of a stream network varies according to the observation scale; the stream network is therefore a multifractal object (Rodriguez-Iturbe \& Rinaldo (1997)). The fractal nature of stream networks could be due to the self-organisation of landscape (Rigon et al. (1994), P. (1996), Rodriguez-Iturbe \& Rinaldo (1997), Dodds \& Rothman (2000)) This means that its characteristics belong to universality classes. Setting up the probability density function of lengths is one of the major issues of hydrology since it allows a precise description of the probability of having a specific distance between the outlet and the segments of stream networks. This function was first developed by Shreve (1969) and known as Width function. It represents a direct connection between the shape, development and hydrological response of a stream network, consequently it has been the subject of many very detailed studies (Gupta \& Mesa (1988), Jin (1992), Rodriguez-Iturbe \& Rinaldo (1997), Gandolfi \& Bischetti (1997)). 


\subsection{Fractals and Morphometry of trees}

Fitter (1982) distinguishes between two classifications that regulate tree structure: one is morphogenetic and follows the example of trees and starts with a single common branch (tree trunk), the other one is morphometric, it follows the example of stream networks and starts with external branches. In his methodological study of the root systems of herbaceous plants, Fitter suggests that Horton's laws may be applied in terms of ordering branches according to the morphometric order, so as to quantify and organise root branching. Holland (1969) shows that the branching pattern of several Eucalyptus species may be described and explained thanks to Horton's laws and the effect of apical dominance on shoot growth.

Leopold (1971) carried out research on several species with different architectures (Abies concolor, Pinus taeda) and reached a similar conclusion. In addition, he claims that the most probable arrangement seems to minimise the total length of twigs in the branching system.

Oohata \& Shidei (1971) use Horton's method to study the branching of four different ligneous plants: evergreen shrubs with broad leaves (Cinnamomum camphora), evergreen conifers, deciduous trees with broad leaves. They show that the branching ratio fluctuates in a much larger extent than stream networks: from 3.0 to 8.0. This ratio varies according to life form.

Withney (1972) shows that the branching ratio of 16 ligneous plants essentially depends on the arrangement of leaves, the deciduous nature of leaves and branches and the size of needles, and that it does not entirely depend on external conditions but is rather specific to one species.

Using Stralher's morphometric tree diagram, Barker et al. (1973) demonstrate 
that in the case of birches and apple trees, logarithms of the average numbers of terminal branches in each branching order, average diameter and number of buds on these branches are lined up with the branching orders on the abscissa. Logarithms of the average lengths of the corresponding branches are much more scattered. They deduce that both species have fractal branching and that lengths are more characteristic of the specific shape of trees. Crawford \& Young (1990) indicate that, in the case of oak trees (Quercus robur), the distribution of branch lengths follows a basic fractal algorithm which can be found in other living organisms. Berger (1991) uses fractals to establish a tree growth scheme (ficus elastica), Chen et al. (1993)) uses them to establish a model of canopy architecture of a poplar stand (Populus sp.) and Mahon \& Kronauer (1976), a model of tree mechanic (Quercus rubra).

Tree structure is still a very topical subject involving intensive research (Sismilich et al. (2003)). Issues involved are multiple, but some of the main areas of interest should be mentioned (Muhar (2001)) : in the field of botany, biomathematic description is a research theme which is strongly encouraged to develop predictive models on plant production, and more particularly on tree diseases. There exists many mathematic models of tree developement and some of them resort to a terminology which is very similar to stream networks, since they deal with plant hydraulics (Niklas (1992), Fruh \& Kurth (1999), Bidel et al. (2000)). In the field of forestry, managing forest development requires that the changes in the shape of trees resulting from the planting conditions (types of soil, co-existence with other species, etc.) be known. It can also help in anticipating the evolution of the forest visual impact and in taking preventive measures (Berezovskaya et al. (1997)). Finally in the field of image processing, working out the most faithful computer simulation of a tree has become 
a central objective especially in terms of commercial software (Viennot et al. (1989)). Whether the methods are determinist such as L-systems (Lidenmayer \& Prusinkiewicz (1988)), stochastic such as the AMAP approach (Jaeger \& de Reffye (1992)) or botanical such as the TREE approach (Bosanac (1990)), the only aim is to obtain the most faithful image of a tree compared to reality.

\subsection{Physical statistics}

Two attempts have been made to apply a physical statistic rationale to hydrography. Lienhard (1964) was the first to become aware of the importance of physical statistics, when applied to rain drops rather than to stream networks. But he simply applied the findings from physical statistics - in this case Maxwell's speed distribution - onto the flow changes after a rainfall. He did not take the characteristics of the drainage area into account, consequently he obtained a model which is neither a forecasting flow model - the only worthwile variable in hydrology - nor a geomorphological model.

Shreve (1966) paved an innovative way when he assumed that the law of stream number of a particular classification order is the result of statistics made on a large number of river segments joining randomly, in the same way as the perfect gas law is the result of statistics of a huge number of molecules colliding randomly. He then undertook to count up directly all the possible combinations, a task which rapidly becomes tedious as the classification order of the network increases. It is obvious that Shreve adopted physical statistics terminology (Shreve (1967)) when he assumed that all stream networks with the same number of headwaters but with different topological characteristics, have the same occurrence probability. Going by a second assumption, also 
used by Smart (1968), he indicated that the lengths of a stream network are independent random variables resulting from one population. Unfortunately, he did not develop this idea through a physical statistics rationale. Several authors will later use these theories on segment distribution, but they will work on assumptions which will not be easily justified (Troutman \& Karlinger (1984)).

As Mandelbrot (1988) explains, we think that when mechanics deals with systems involving a great number of molecules, laws governing influences at the local level are known with great accuracy, but their interaction at the global level remains little known. The situation is worse when it comes to geomorphology, since local and global levels are both unknown. Therfore, in geomorphology more so than in mechanics, the solution should be statistical. The physician's concept of chance has been shaped by quantum mechanics and thermodynamics. In both theories, chance occurs at the microscopic level where it plays an essential role, whereas at the microscopic level, its role is a very minor one. Conversely, concerning the objects we are studying, internal homothecy gives chance precisely the same importance at both levels and speaking of the microscopic or macroscopic levels consequently becomes irrelevant.

Actually, it seems that in the field of geomorphology, only a statistical approach can be productive. According to Diu et al. (1995), physical statistics aims to explain the properties of bodies on a standard scale (macroscopic dimension) using the properties of microscopic constituents. Nevertheless, since geomorphology does not make a clear separation of the order of magnitude between the microscopic dimension and the macroscopic one, physical statistics rationales could be applied to this area of research, provided physical 
statistics is assigned a broader objective: to explain the properties of complex systems such as stream networks starting from the properties of their elementary constituents, though not necessarily microscopic ones. Nevertheless, it is necessary to make sure that two conditions which implicitely constitute the foundation of physical statistics are fulfilled:

- compared to the analysed constituents, the system should be very large;

- localised properties of the system should be fairly homogeneous.

The limits of validity of the law we are now going to establish are probably closely linked to the fulfilment of both conditions.

\section{The model}

The model introduced here uses findings of research carried out on stream networks, and applies them to trees, and more specifically to their branching structures.

The focus is to describe tree structure thanks to Strahler stream ordering (1964), and to apply Horton's laws (1945) to a physical statistics rationale, in order to elaborate a descriptive theoretical model of branch lengths.

\subsection{Tree structure and ordering}

The fundamental difference between studies carried out on stream networks and the tree structural model proposed here, lies in the fact that the tree is analysed in three dimensions, whereas stream networks are projected on to a plane. Nevertheless, both approaches (Strahler stream ordering, Horton's 
laws) to analyse stream networks in two dimensions can be applied directly to a three-dimensional tree modelling.

The analogy with stream networks entitles us to assert that a branch (likened to a segment) is an element of a tree situated between two successive branching. Consequently, Strahler stream ordering can be implemented:

- A bud or a terminal branch belong to the first order;

- Two order $i$ branches form an order $i+1$ branch;

- When two branches in different orders join, they form a branch which is assigned the larger order.

The tree trunk (likened to the outlet) is assigned the larger order, corresponding to the order of the tree.

Horton's laws also make it possible to work out the $R_{B}$ and $R_{L}$ :

$$
\begin{aligned}
R_{L} & =\frac{\overline{l_{k}}}{\overline{l_{k-1}}} \\
R_{B} & =\frac{N_{k-1}}{N_{k}}
\end{aligned}
$$

Where $\overline{l_{k}}$ is the average value of the morphometric lengths of $k$ order and $N_{k}$ is the number of morphometric lengths of $k$ order.

\subsection{Definition of morphometric length}

Before giving the theoretical expression of probability density function of morphometric lengths, it is necessary to define this variable accurately. As shown in Figure 3, if we take an indefinite point on one branch, the path to be covered between this point and the trunk collar successively goes over branches 
of increasing orders. The morphometric length is defined as follows:

$$
L=\sum_{k=1}^{n} l_{k}
$$

$l_{k}$ is the length of the branch or branch segment in $k$ order and $n$ is the order of the tree. Hence, the morphometric length is the added lengths $n$ of the branches.

The morphometric length $L$ may be calculated using a vector with $n$ components $\left(l_{1}, l_{2}, \cdots, l_{n}\right)$.

\subsection{Choosing a symbolic space}

Let us have a symbolic space, named morphometric lengths space. This symbolic space has $n$ dimensions corresponding to $n$ axis onto which $n$ components of morphometric lengths are projected. Figure 3 is a specific example for $k=n=3$. Then the $\left(l_{1}, l_{2}\right.$ et $\left.l_{3}\right)$ three components of $L$ can be projected onto the axis of the symbolic space which is only three-dimensional in this case.

Let us consider an elementary volume $d_{l}=\left(d l_{1}, d l_{2}, \cdots, d l_{n}\right)$, ), the probability of having a morphometric length between $\left(l_{1}, l_{2}, \cdots, l_{n}\right)$ and $\left(l_{1}+d l_{1}, l_{2}+d l_{2}, \cdots\right.$, $\left.\left.n+d l_{n}\right)\right)$ volume interval is $F\left(l_{1}, l_{2}, \cdots, l_{n}\right) d l_{1}, d l_{2}, \cdots, d l_{n}$.

Where $F$ is the probability density function of $L=\left(l_{1}, l_{2}, \cdots, l_{n}\right)$. If we have $d^{n} N$, the number of morphometric lengths between $\left(l_{1}, l_{2}, \cdots, l_{n}\right)$ and $\left(l_{1}+d l_{1}\right.$, $\left.l_{2}+d l_{2}, \cdots, l_{n}+d l_{n}\right)$ volume interval, then:

$$
F\left(l_{1}, l_{2}, \cdots, l_{n}\right) d l_{1} d l_{2} \cdots d l_{n}=\frac{d^{n} N}{N}
$$

Where $N$ is the total number of morphometric lengths. 
At this stage of the rationale, we must take the self-similarity properties of our branching system into account, and use one of Horton's laws. Beside the length ratio $\left(R_{L}\right)$ defined in this particular law, the following ratio may be given:

$$
r=\frac{l_{k}}{l_{k-1}}
$$

In fact Duchesne et al. (1997) and Cudennec (2000) demonstrate that $r \simeq R_{L}$.

To explain the form of the function $F$ with more accuracy, we should make working hypotheses.

\subsection{Choosing the fundamental hypothesis}

- The independence hypotheses of the distribution function of components reduced by the morphometric length. According to this hypothesis, the distribution function of a component $l_{k}$ only depends on $l_{k}$, not on any of the other components $l_{j}, k$ being different from $j$. This leads us to give an expression of fonction $F$ as a product of $n$ fonctions for one variable:

$$
F\left(l_{1}, l_{2}, \cdots, l_{n}\right)=f_{1}\left(l_{1}\right) f_{2}\left(l_{2}\right) \cdots f_{n}\left(l_{n}\right)
$$

- The isotropy hypothesis of distributions. We consider that the distribution law of $l_{k}$ is isotropic. Because of the self-similarity nature of tree structure and Horton's law, the $k$ order component is one factor $r$ larger on average than the $k-1$ order component. The isotropy hypothesis should then be applied, with the reduced components of branch lengths $\frac{l_{k}}{r^{i-1}}$ as coordinates of the symbolic space, rather than the components $l_{k}$.

For calculation reasons, we will extract the square root of these reduced com- 
ponents:

$$
z_{k}=\sqrt{\frac{l_{k}}{r^{k-1}}}=\frac{x_{k}}{r^{\frac{k-1}{2}}}
$$

The isotropy hypothesis helps consider all functions $f_{k}\left(l_{k}\right)$ identical in relation (6):

$$
F\left(l_{1}, l_{2}, \ldots, l_{n}\right)=f\left(l_{1}\right) f\left(\frac{l_{2}}{r}\right) \ldots f\left(\frac{l_{n}}{r^{n-1}}\right)
$$

then

$$
\phi\left(z_{1}, z_{2}, \ldots, z_{n}\right)=\varphi\left(z_{1}\right) \varphi\left(z_{2}\right) \ldots \varphi\left(z_{n}\right)
$$

Where functions $\phi$ and $\varphi$ are respectively different from functions $F$ and $f$.

Function $\phi$ can also be defined as:

$$
\phi\left(z_{1}, z_{2}, \ldots, z_{n}\right)=\frac{d^{n} N}{N d z_{1} d z_{2} \ldots d z_{n}}
$$

The isotropy hypothesis means that the density of points representing the extremities of vectors in the symbolic space has a spherical symmetry. The reduced components $z_{k}$ represent a solution to the equation of a hypersphere:

$$
z_{1}^{2}+z_{2}^{2}+\ldots+z_{n}^{2}=C^{s t e}
$$

The distribution function of $z_{k}$ should then have a constant value:

$$
\phi\left(z_{1}, z_{2}, \ldots, z_{n}\right)=C^{s t e}
$$

Both hypotheses allow us to determine the probability density function of morphometric lengths. 
2.5 Determination of the probability density function of reduced morphometric lengths

Derivating relations (11) and (12), we have:

$$
2 z_{1} d z_{1}+2 z_{2} d z_{2}+\ldots+2 z_{n} d z_{n}=0
$$

From which it follows:

$$
\frac{\partial \phi}{\partial z_{1}} d z_{1}+\frac{\partial \phi}{\partial z_{2}} d z_{2}+\ldots+\frac{\partial \phi}{\partial z_{n}} d z_{n}=0
$$

According to relation (9) :

$$
\frac{1}{\phi} \frac{\partial \phi\left(z_{k}\right)}{\partial z_{k}}=\frac{1}{\varphi\left(z_{k}\right)} \frac{d \varphi\left(z_{k}\right)}{d z_{k}} \quad \forall i, 1 \leq i \leq n
$$

So (12) can be replaced with:

$$
\frac{1}{\varphi\left(z_{1}\right)} \frac{d \varphi\left(z_{1}\right)}{d z_{1}}+\frac{1}{\varphi\left(z_{2}\right)} \frac{d \varphi\left(z_{2}\right)}{d z_{2}}+\ldots+\frac{1}{\varphi\left(z_{n}\right)} \frac{d \varphi\left(z_{n}\right)}{d z_{n}}=0
$$

This mathematical expression helps determine the various values $z_{1}, z_{2}, \ldots, z_{n}$ where function $\varphi$ reaches an extremum value. If there is no restrictive condition for this equation, we can demonstrate that a solution can be obtained by cancelling all the derivatives of the function.

Still, equation (11) precisely expresses a restrictive relation on the $z_{k}$ components. This constraint is handled by using Lagrange multipliers method (Sears (1971)), relations (11) and (16) can then be combined, multiplying (11) by a constant value $\lambda$ and adding each term to relation (16). This results in the following equation, in which $n$ differentials are considered to be independent:

$$
\left[2 \lambda z_{1} \frac{1}{\varphi\left(z_{1}\right)} \frac{d \varphi\left(z_{1}\right)}{d z_{1}}\right] d z_{1}+\ldots+\left[2 \lambda z_{n} \frac{1}{\varphi\left(z_{n}\right)} \frac{d \varphi\left(z_{n}\right)}{d z_{n}}\right] d z_{n}=0
$$


Only then can we consider differentials to be independent, and the solution to equation (17) amounts to cancelling each differential successively:

$$
\frac{1}{\varphi\left(z_{k}\right)} \frac{d \varphi\left(z_{k}\right)}{d z_{k}}=-2 \lambda z_{k} \quad \forall i, 1 \leq i \leq n
$$

Equation (18) is successively equal to:

$$
\begin{aligned}
\frac{d \log \left(\varphi\left(z_{k}\right)\right)}{d z_{k}} & =-2 \lambda z_{k} \quad \forall i, 1 \leq i \leq n \\
\log \left(\varphi\left(z_{k}\right)\right) & =-\lambda z_{k}^{2}+C^{s t e} \quad \forall i, 1 \leq i \leq n \\
\varphi\left(z_{k}\right) & =A e^{-\lambda z_{k}^{2}} \quad \forall i, 1 \leq i \leq n
\end{aligned}
$$

Fonction $\varphi$, is a normal distribution with a zero mean and a standard deviation $\frac{1}{\sqrt{2 \lambda}}$. Constante $A$ can be calculated since $\varphi$ is a probability density function, consequently by definition, its integral taken between $-\infty$ and $\infty$ should be equal to 1:

$$
\int_{-\infty}^{\infty} \varphi\left(z_{k}\right) d z_{k}=\int_{-\infty}^{\infty} A e^{-\lambda z_{k}^{2}} d z_{k}=1
$$

The solution to this integral is definitely known (Spiegel (2000)) and gives to $A$ a value of $\sqrt{\frac{\lambda}{\pi}}$. Therefore, function $\varphi$ is known to the very $\lambda$ Lagrange multiplier, and by modifying the variable of relation (7), we have the probability density function of reduced components of branch lengths:

$$
f d p\left(l_{k}\right)=\frac{1}{\sqrt{\pi}} \sqrt{\frac{\lambda}{r^{i-1}}} \frac{1}{\sqrt{l_{k}}} e^{-\frac{\lambda l_{k}}{r^{i-1}}}
$$

Moreover, constant $\lambda$ may be explained by expressing the $l_{k}$ components average, named $\overline{l_{k}}$, it gives successively:

$$
\overline{l_{k}}=\int_{0}^{\infty} l_{k} f d p\left(l_{k}\right) d l_{k}
$$




$$
\begin{aligned}
& =\frac{1}{\sqrt{\pi}} \sqrt{\frac{\lambda}{r^{k-1}}} \int_{0}^{\infty} \frac{l_{k}}{\sqrt{l_{k}}} e^{-\frac{\lambda l_{k}}{r^{k-1}}} d l_{k} \\
& =\frac{1}{\sqrt{\pi}} \sqrt{\frac{\lambda}{r^{k-1}} \int_{0}^{\infty} l_{k}^{\frac{1}{2}} e^{-\frac{\lambda l_{k}}{r^{k-1}}} d l_{k}} \\
& =\frac{1}{\sqrt{\pi}} \sqrt{\frac{\lambda}{r^{k-1}} \Gamma\left(\frac{1}{2}+1\right)\left(\frac{r^{k-1}}{\lambda}\right)^{\frac{3}{2}}} \\
& =\frac{r^{k-1}}{2 \lambda}
\end{aligned}
$$

After replacing this result in equation (23) we have:

$$
f d p\left(l_{k}\right)=\frac{1}{\sqrt{2 \pi \overline{l_{k}}}} \frac{1}{\sqrt{l_{k}}} e^{-\frac{l_{k}}{2 \bar{l}_{k}}}
$$

A Gamma distribution with $\alpha=\frac{1}{2}$ and $\beta=2 \overline{l_{k}}$ can be recognised here and is named $\Gamma\left(\frac{1}{2}, 2 \overline{l_{k}}\right)$.

The second step consists of determining the probability density distribution for $L$, knowing that $L=\sum_{k=1}^{n} l_{k}$. The most usual way to calculate the distribution $p d f(L)$, is to use the following property: If we have $n$ independent random variables $l_{k}, k=1,2, \ldots, n$ of probability density function $f d p\left(l_{k}\right)$ then the random variable $L$ defined as $L=\sum_{k=1}^{n} l_{k}$ has a probability density function $p d f(L)$ such as:

$$
p d f(L)=p d f\left(l_{1}\right) \star p d f\left(l_{2}\right) \star \ldots p d f\left(l_{n}\right)
$$

Where $\star$ is the convolution integral. 


\subsection{Convolution integral of two Gamma distributions}

Let us work out the convolution integral of two Gamma distributions with $\beta$ parameters which are different in pairs, we then try to calculate $\Gamma\left(\alpha, \beta_{1}\right) \star$ $\Gamma\left(\alpha, \beta_{2}\right)$. To do this calculation, we will use the characteristic Gamma distribution functions. In fact, to convolute both Gamma distributions means that we are looking for the probability density function $p d f(L)$ for $L$ such as $L=l_{1}+l_{2}$ where $l_{1}$ and $l_{2}$ have a probability density function represented by the functions $\Gamma\left(\alpha, \beta_{1}\right)$ and $\Gamma\left(\alpha, \beta_{2}\right)$. Moreover, the characteristic functions, named $\varphi_{l_{k}}^{\star}$, have the following property:

$$
\varphi_{l_{1}+l_{2}}^{\star}=\varphi_{l_{1}}^{\star} \varphi_{l_{2}}^{\star}
$$

The characteristic function of a random variable, named $l_{k}$ variables here, is defined as a mathematical expectation of the exponential function of this random variable; in the present case we have therefore:

$$
\begin{aligned}
\varphi_{l_{k}}^{\star}(s) & =E\left[e^{-s l_{k}}\right] \\
& =\int_{0}^{\infty} e^{-s l_{k}} f d p\left(l_{k}\right) d l_{k} \\
& =\frac{1}{\Gamma(\alpha) \beta_{k}^{\alpha}} \int_{0}^{\infty} l_{k}^{\alpha-1} e^{-l_{k}\left(s+\frac{1}{\beta_{k}}\right)} d l_{k} \\
& =\frac{1}{\left(1+s \beta_{k}\right)^{\alpha}}
\end{aligned}
$$

Here on have:

$$
\varphi_{l_{1}+l_{2}}^{\star}(s)=\frac{1}{\left[\left(1+s \beta_{1}\right)\left(1+s \beta_{2}\right)\right]^{\alpha}}
$$


Concerning our case, $\alpha=\frac{1}{2}$, then function $p d f(L)$ such as $L=l_{1}+l_{2}$ can be worked out thanks to Laplace transform tables:

$$
p d f(L)=\frac{1}{\sqrt{\beta_{1} \beta_{2}}} e^{-\frac{\left(\frac{1}{\beta_{1}}+\frac{1}{\beta_{2}}\right) L}{2}} I_{0}\left(\frac{\left(\frac{1}{\beta_{2}}-\frac{1}{\beta_{1}}\right) L}{2}\right)
$$

Where $I_{0}(x)=1+\frac{x^{2}}{2^{2}}+\frac{x^{4}}{2^{2} 4^{2}}+\frac{x^{6}}{2^{2} 4^{2} 6^{2}}+\ldots$ is the modified Bessel function of the first kind and zero order. The result of the probability density function of morphometric lengths $L$ is therefore:

$$
p d f(L)=\frac{1+r}{2 \sqrt{r} \bar{L}} e^{-\frac{(1+r)^{2} L}{4 r \bar{L}}} I_{0}\left(\frac{(1-r)(1+r) L}{4 r \bar{L}}\right)
$$

\subsection{Convolution integral of $n$ Gamma distributions}

The result of the convolution integral for $n$ Gamma distributions is obtained by working out the generalised characteristic function(Mathai (1982), Moschopoulos (1985), Sim (1992)) and we can thus assert the following theorem:

Let $X_{1}, X_{2}, \ldots, X_{n}, n$ be $n$ independent random distributed according to a Gamma distribution of the following type:

$$
\Gamma\left(x, \alpha_{i}, \beta_{i}\right)=\frac{\beta_{i}^{\alpha_{i}} x^{\alpha_{i}-1}}{\Gamma\left(\alpha_{i}\right)} e^{-\beta_{i} x}
$$

Then the probability density for $Y=\sum_{i=1}^{n} X_{i}$, is:

$$
p d f(y)=\left(\prod_{i=1}^{n} \beta_{i}^{\alpha_{i}}\right) \frac{y_{i=1}^{n-1} \alpha_{i}-1}{\Gamma\left(\sum_{i=1}^{n} \alpha_{i}\right)} e^{-\beta_{n} y} \sum_{k=0}^{\infty} \frac{b_{n}(k)\left(\sum_{i=1}^{n-1} \alpha_{i}\right)_{k}}{k !\left(\sum_{i=1}^{n} \alpha_{i}\right)_{k}}\left[\left(\beta_{n}-\beta_{n-1}\right) y\right]^{k}(30)
$$


where

$$
b_{i}(k)=\left\{\begin{array}{lr}
1, & \text { if } i=2 \\
\sum_{j=0}^{k} \frac{b_{i-1}(j)\left(\sum_{p=1}^{i-2} \alpha_{p}\right)_{j}(-k)_{j}}{j !\left(\sum_{p=1}^{i-1} \alpha_{p}\right)_{j}} C_{i}^{j} \text { for } i=3,4, \ldots, n
\end{array}\right.
$$

With

$$
C_{i}=\frac{\beta_{i-2}-\beta_{i-1}}{\beta_{i}-\beta_{i-1}}
$$

and

$$
(x)_{k}=x(x+1)(x+2) \ldots(x+k-1)
$$

By returning to our initial notations, the probability density function of morphometric lengths is defined by the following expression:

$$
p d f(L)=\left(\frac{\sum_{i=1}^{n} r^{i-\frac{n+1}{2}}}{2 \bar{L}}\right)^{\frac{n}{2}} \frac{L^{\frac{n}{2}-1}}{\Gamma\left(\frac{n}{2}\right)} e^{-\frac{\sum_{i=1}^{n} r^{i-n} L}{2 \bar{L}}} \sum_{k=0}^{\infty} \frac{b_{n}(k)\left(\frac{n-1}{2}\right)_{k}}{k !\left(\frac{n}{2}\right)_{k}}\left(\frac{1-r^{n}}{2 \bar{L} r^{n-1}} L\right)^{k}
$$

and

$$
b_{i}(k)=\left\{\begin{array}{lr}
1, & \text { if } i=2 \\
\sum_{j=0}^{k} \frac{b_{i-1}(j)\left(\frac{i-2}{2}\right)_{j}(-k)_{j}}{j !\left(\frac{i-1}{2}\right)_{j}}(-r)^{j} & \text { for } i=3,4, \ldots, n
\end{array}\right.
$$




\section{Applications to Cupressocyparis}

The only way of testing our theoretical model is to measure the morphometric lengths of a tree. To do so, we have chosen Cupressocyparis leylandii, (see photos 4), a conifer which can grow up to several tens of meters high, and which is often mistaken for Thuya plicata because both are currently used for trimmed hedges. We have chosen a young tree (3 years) about one meter high.

To determine the morphometric lengths of this tree means measuring distances between its basis and all apexes. We have chosen a young tree in order to restrict the measurements.

First we have cut up the tree (see photos 4) and classified the branches to keep the tree structure. Then we have taken 7490 measurements manually to determine morphometric lengths. A concise account of measurements is given in table 2. It includes the number of $k$ order lengths, the average lengths for each $k$ and the corresponding Horton's ratios $\left(R_{L}\right.$ and $\left.R_{B}\right)$.

It can be noticed that $R_{L}$ ratios are lower in average than stream networks'ratios, whereas this not the case with $R_{B}$ ratios (according to the Oohata \& Shidei (1971)'s results). The high average values of $R_{B}$ reveal that there is a larger number of branching in $k+1$ order than $k$ order. In fact, there are 7488 first order branchings compared to 1608 second order branchings and so forth. Concerning the $R_{L}$ ratio, its low values compared to those of stream networks, show that the average lengths do not differ extremely from one order to another. 
Once the morphometric lengths have been captured digitally, it is easy for us to determine their distribution, that is to say the histogram of the tree morphometric lengths. The borderline case histogram - when the class interval approaches 0 - is a probability density function $(p d f)$. Then this experimental histogram may be compared with the theoretical law (33) which is in fact the probability density function of morphometric lengths.

Our theoretical model (equation 33) requires three variables: Strahler ordering $n$, the average of morphometric lengths $\bar{L}$ and Horton's ratio $r$. But considering the variations in the ratio (see Table 2), which value should be chosen? It should be remembered that in the demonstration section (see equation 5), Horton's ratio $r$ is defined as the ratio of successive order components rather than average components, as this is usually the case with stream networks. This definition, along with our isotropy hypothesis has led us to establish the probability density function of morphometric lengths $l_{k}$ given in equation (24). To calculate the value $r$ means assessing the optimal reduction factor, so that the histograms of $\frac{l_{k}}{r^{k-1}}$ are as similar as possible. This is shown in Figure 5, as well as the morphometric lengths histograms of orders 1 to 5, before (Figure 5 a) and after (Figure $5 \mathrm{~b}$ ) reduction with factor $r$.

A value of $r=1.5$ is found for Horton's ratio; this value allows $\frac{l_{k}}{r^{k-1}}$ histograms to be as superimposed as possible and it offers complete validation to our isotropy hypothesis.

It is obvious that no factor $r$ may adjust the 5 histograms accurately and this is where our isotropy hypothesis reaches its limits. Indeed, this is the only difference between self-similar fractal mathematics with infinite order and nat- 
ural fractal structures whose self-similarity is no longer obvious beyond some order, which is often very small $(n=2$ or 3$)$.

With this value $r$, we have all variable values for our model:

- $n=5$, for the Strahler hierarchical ordering of the studied tree;

- $\bar{L}=163.7 \mathrm{~mm}$, for the average of morphometric total lengths;

- $r=1.5$, for Horton's ratio.

Our model can now be compared with the experimental histogram of morphometric lengths, as shown in Figure 6. It can be noticed that the histogram of the morphometric lengths which have been measured on the tree and the theoretical law (equation 33) adjust almost perfectly. It is essential to add that the adjustment does not require any fitting, since the variable values (appearing above) result from measurements on the tree; they are consequently a necessary and sufficient condition to put forward equation (33).

This theoretical model not only simulates properly the allometry of the studied tree, but also the relation which exists between the morphometric lengths and the tree structure. We are tempted to think that this simulated relation can be found in many tree species (forthcoming research on apple trees) as shown for example on photo 8 representing a limetree showing clearly that the distribution of apexes is more important near the tree base. 


\section{Discussion et conclusion}

The plant stem only grows at the first millimeters or centimeters level of stem extremity, also called the apex. Photo 8 shows that the maximum number of apexes is to be found slightly above plant base; beyond this point, the number of apexes decreases very gradually. Branching points come out on the apex. Trees have two branching patterns depending on species (Hallé (1970)). In the case of the continuous growth type, lateral branching comes out slightly behind the apex, as the axis keeps on growing (Figure 7 a). This is the case of Cupresocyparis leylandii. Concerning the rythmic growth type, branching only comes out after a dormant period of several weeks or months of the main axis ; then when the growth period starts again, branching occurs where growth has stopped (Figure $7 \mathrm{~b}$ ); this is the case of the apple tree Malus pumila, submitted in a previous publication (Duchesne et al. (2002)). The Gamma law applies to both branching patterns, although they are completely different.

The apex of the main stem causes inhibition of the secondary apexes and exerts growth control, a common phenomenon in branched plants named apical dominance by biologists. In young trees, apical dominance is exerted in succession on 5 - 7 branching orders, which are organised into a hierarchy. As the plant grows, the main axis loses control of the branching, and then the tree includes a set of several hierarchical systems which are more or less independent from one another on the biological level (Raimbault \& Tanguy (1993) and Raimbault et al. (1995)). Visually, this evolution results in the withdrawal of the main axis to the benefit of several equivalent axes (Figure $7 \mathrm{c}$ ). This biological evolution results in a drift of the height of the curve representing 
the Gamma law towards the right, which tends to a Gaussian distribution (forthcoming publication on apple trees).

What is the point of such a mathematical study?

If the branched shapes of trees are now described precisely, the genesis of such shapes poses insoluble problems. Hierarchical branching of apical dominance through gradient and flow of hormone distribution is now understood quite well (Cline (1994) and Cline (1997)). But this does not account for the complexity of branching. Studying the distribution of apexes morphometric lengths should enhance discussion on the causality of plant morphogenesis. Basically, do plants branch in response to an internal impulse, as viscous fingering patterns are obtained by injecting between two blades poorly viscous fluid into a more viscous fluid?

Or does branching result from an external attraction from the environment? It is likely that branching is the result of a strong interference between several processes belonging to both categories.

Measuring morphometric lengths of trees and interpreting their structure is arduous delicate work. Obviously, the research we have carried out on our young Cupressocyparis proves to be impossible to do on an oak tree several meters high. Consequently, we have developed a protocole of photogammetric measures in order to make the task easier. Photogammetry helps the 3-D reconstruction of complex objects, thanks to the processing of digital photographs. 


\section{References}

Abrahams, A. (1984). Channel networks : a geomorphological perspective. Water Resources Res., (20), 161-168.

Barbera, P. L. \& Rosso, R. (1987). Fractal geometry of river networks. EOS Transaction AGU, 68, 12-76.

Barbera, P. L. \& Rosso, R. (1989). On the fractal dimension of stream networks. Water Resources Res., 25(4), 735-741.

Barbera, P. L. \& Rosso, R. (1990). On the fractal dimension of stream networks. Water Resources Res., 26, 2245-2248.

Barker, S., Cumming, G., \& Horsfield, K. (1973). Quantitative morphometry of the branching structure of trees. J. Theor. Biol., 40, 33-43.

Barnsley (1988). Fractals everywhere. Academic Press.

Barton, C. (1995). Fractals in the earth sciences. Hardcover.

Berezovskaya, F., Karev, G., Kisliuk, O., Khlebopros, R., \& Tcelniker, Y. (1997). Fractal approach to computer-analytical modeling of tree crown. J. Trees, 11, 323-327.

Berger, D. (1991). Modification of a simple fractal tree growth scheme: implications on growth, variation and evolution. J. Theor. Biol., 152, 513-529.

Bidel, L., Pagès, L., Rivière, L., Pellous, G., \& Lorendeau, J. (2000). Massflowdyn i: a carbon transport and partitioning model for root system architecture. Annals of botany, 85, 869-886.

Bosanac, B. (1990). Tree: a knowledge-based system for generating images of trees. Graduate school of design.

Chen, S., Impens, I., Ceulemans, R., \& Kochelbregh, F. (1993). Measurement of gap fraction of fractal generated canopie using digitalized image analysis. Agricultural and Forest Meteorology, 65, 245-259. 
Cline, M. (1994). The role of hormones in apical dominance. new approaches to an old problem in plant development. Physiologia plantarum, 90, 230-237.

Cline, M. (1997). Concepts and terminology of apical dominance. American Journal of Botany, 84(8), 1064-1069.

Crawford, J. \& Young, I. (1990). A multiple scaled fractal tree. J. Theor. Biol., 145, 199-206.

Cudennec, C. (2000). Description mathématique de l'organisation du réseau hydrographique et modélisation hydrologique. Thèse de Doctorat, ENSAR, Rennes.

Dauphiné, A. (1995). Chaos, fractales et dynamiques en géographie. Reclus : "espaces modes d'emploi".

Dekking (1999). Fractals : theory and applications in engineering. Michel.

Diu, B., Guthmann, D., Lederer, D., \& Roulet, B. (1995). Eléments de physique statistique. Hermann.

Dodds, P. \& Rothman, D. (2000). Scaling, universality and geomorphology. Ann. Rev. Earth Planet. Sci., 28, 571-610.

Dooge, J. (1986). Looking for hydrological laws. Water Resources Res., 22, 46S-58S.

Douady, S. \& Couder, Y. (1992). Phyllotaxis as a physical self-organized growth process. Physical Review Letters, 68(13), 2098-2101.

Duchesne, J., Cudennec, C., \& Corbierre, V. (1997). Relevance of the h2u model to predict the discharge of a catchment. Water Science and Technology, 36, 169-175.

Duchesne, J., Raimbault, P., \& Fleurant, C. (2002). Towards a universal law of tree morphometry by combining fractal geometry and statistical physics. Proceeding: Emergent Nature, Patterns, growth ans scaling in the sciences, pages $93-102$. 
Feder, J. (1988). Fractals. Plenum Press.

Fitter, A. (1982). Morphometric analysis of root systems: application of the technic and influence os soil fertility on root system development in two herbaceous species. Plant Cell and Environment, 5, 313-322.

Frankhauser, P. (1994). La fractalité des structures urbaines. Anthropos.

Fruh, T. \& Kurth, W. (1999). The hydraulic system of trees: theorical framework and numerical simulation. J. Theor. Biol., 201, 251-270.

Gandolfi, C. \& Bischetti, G. (1997). Influence of the drainage network identification method on geomorphology properties and hydrological response. Hydrological Processes, 11, 353-375.

Gouyet, J. (Physique et structures fracales). 1992. Masson.

Gupta, V. \& Mesa, O. (1988). Runoff generation and hydrologic response via channel network geomorphology-recent progress and open problems. 102, $3-28$.

Hallé, F. (1970). Essai sur l'architecture et la dynamique de croissance des arbres tropicaux. Masson.

Holland, P. (1969). The maintenance of structure and shape ogf three mallee eucalypts. New Phytol., 68, 411-421.

Horton, R. (1945). erosional development of streams and their drainage basins; hydrological approach to quantitative morphology. Bulletin of the Geological Society of America, 56, 275-370.

Jaeger, M. \& de Reffye, P. (1992). Basic concepts of computer simulation of plant growth. in: The 1990 mahabaleshwar seminar on modern biology, mahabaleshwar (ind). J. Biosciences, 17, 275-291.

Jin, C. (1992). A deterministic gamma-type geomorphologic instantaneous unit hydrograph based on path types. Water Resources Res., 28(2), 479486. 
Kirshen, D. \& Bras, R. (1983). The liear channel and its effects on the geomorphological iuh. J. Hydrology, 65, 175-208.

Korvin, G. (1992). Fractal models in earth sciences. elsevier.

Leopold, L. (1971). Trees and streams: the efficiency of branching patterns. J. Theor. Biol., 31, 339-354.

Lidenmayer, A. \& Prusinkiewicz, P. (1988). Development models of multicellular organism: a computer graphics perspective. in langton ec (ed.) artificial life.

Lienhard, J. (1964). A statistical mechanical prediction of the dimensionless uh. J. Geophys. Res., 69, 5231-5238.

Mahon, T. M. \& Kronauer, R. (1976). 1976. J. Theor. Biol., 59, 443-466.

Mandelbrot, B. (1988). Fractal geometry of nature. Hardcover.

Mandelbrot, B. (1995). Les objets fractals. Flammarion.

Mathai, A. (1982). Storage capacity of a dam with gamma type inputs. Ann. Inst. Statist. Math., 34, 591-597.

Moschopoulos, P. (1985). The distribution of the sum of independent gamma random variables. Ann. Inst. Statist. Math., 37, 541-544.

Muhar, A. (2001). Three-dimensional modelling and visualisation of vegetation for landscape simulation. Landscape and Urban Planning, 54, 5-17.

Nakagawa, M. (1999). Chaos and fractals in engineering. World Scientific Publishing Company.

Niklas, K. (1992). Plant biomechanic: an engineering approach to plant form and function. The University of Chicago Press.

Nikora, V. (1991). Fractal structures of river plan forms. Water Resources Res., 27(6), 1327-1333.

Nikora, V. \& Sapozhnikov, V. (1993). River network, fractal geometry and its computer simulation. Water Resources Res., 29(10), 3569-3575. 
Nikora, V., Sapozhnikov, V., \& Noever, D. (1993). Fractal geometry of individual river channels and its computer simulation. Water Resources Res., 29(10), 3561-3568.

Oohata, S. \& Shidei, T. (1971). Studies on the branching structure of trees. Japonese Journal of Ecology, 217(1-2), 7-14.

P., B. (1996). How nature Works ? Copernicus/Springer-Verlag.

Peitgen H., H. J. \& Saupe, D. (1992). Fractals for the classroom. SpringerVerlag.

Raimbault, P. \& Tanguy, M. (1993). La gestion des arbres d'ornement. 1 partie : Une methode d'analyse et de diagnostic de la partie aeŕienne. Revue Forestière Française, 2, 97-116.

Raimbault, P., de Jonghe, F., \& andM. Tanguy, R. T. (1995). La gestion des arbres d'ornement. 2 partie : Gestion de la partie aeŕienne : les principes de la taille longue moderne des arbres d'ornement. Revue Forestière Française.

Rigon, R., Rinaldo, A., \& Rodriguez-Iturbe, I. (1994). On landscape selforganization. J. Geophys. Res., 99(11), 971-993.

Rodriguez-Iturbe, I. \& Rinaldo, A. (1997). Fractal river basins. Cambridge University Press.

Roland, B. \& Fleurant, C. (2002). Characterization of hedgrow lattice by means of fractal geometry. submited in october 2002.

Rosso, R., Bacchi, B., \& Barbera, P. L. (1991). Fractal relation of mainstream length to catchment area in river networks. Water Resources Res., 27(3), $381-387$.

Sapoval, B. (1997). Universalités et fractales. Flammarion.

Schroeder (1991). Fractals, chaos, power laws. Freeman ans Company.

Schumm, S. (1956). Evolution of drainage system and slopes in badlands at pearth amboy. Bulletin of the Geological Society of America, 87, 597-646. 
Sears, F. (1971). An introduction to thermodynamics, the kinetic theory of gases, and statistical mechanics. Addison-Wesley.

Shreve, R. (1966). Statistical law of stream numbers. Journal of Geology, 74, $17-37$.

Shreve, R. (1967). Infinite topologically random channel networks. Journal of Geology, 75, 178-186.

Shreve, R. (1969). Stream lengths and basin areas in topologically random channel networks. Journal of Geology, 77, 397-414.

Sim, C. (1992). Point processes with correlated gamma interarrival times. Statistics and Probability Letters, 15, 135-141.

Sismilich, M., Menzies, M., Gandar, P., Jameson, P., \& Clemens, J. (2003). Development of a mathematical method for classifying and comparing tree architecture using parameters from a topological model of a trifurcating botanical tree. J. Theor. Biol., 220, 371-391.

Smart, J. (1968). Statistical properties of stream lengths. Water Resources Res., 4, 1001-1014.

Spiegel, M. (2000). Mathematical Handbook. Schaum's outline series.

Strahler, A. (1957). quantitative analysis of watershed geomorphology. Transaction $A G U, \mathbf{3 8}, 913-920$.

Tarboton, D., Bras, R., \& Rodriguez-Iturbe, I. (1988). The fractal nature of river networks. Water Resources Res., 25(8), 1317-1322.

Tarboton, D., Bras, R., \& Rodriguez-Iturbe, I. (1990). Comment on "on the fractal dimension of stream networks" by p. la barbera and r. rosso. Water Resources Res., 26, 2243-2244.

Troutman, B. \& Karlinger, M. (1984). On the expected width function for topologically random channel networks. J. Applied Probability, 21, 836-849.

Turcotte, D. (1992). Fractals and chaos in geology and geophysics. Cambridge 


\section{University Press.}

Vicsek (1994). Fractal in natural sciences. Hardcover.

Viennot, X., Eyrolles, G., Janey, N., \& Arquès, D. (1989). Combinatorial analysis of ramified patterns and computer imagery of trees. Computer Graphics, 23(3), 31-40.

West, G., Brown, J., \& Enquist, B. (1997). A general model for the origin of allometric scaling laws in biology. Science, 276, 122-126.

Withney, G. (1972). The bifurcation ratio as an indicator of adaptative strategy in woody plant species. Bull. of the Torrey Botanical Club, 103(2), $67-72$. 


\section{Figures and Tables}

Figure 1 : Left side, Cupressocyparis structure; right side, river Loire (France) structure.

Figure 2 : The concept of Stralher's hierarchical classification system.

Figure 3 : The morphometric length is the added lengths of branches up to an indefinite point of the tree. Here, the path between an apex of the tree and the trunk basis.

Figure 4 : The Cupressocyparis is first cut up and then is classified to keep its structures.

Figure 5 : Experimental histogramms of $l_{k}$ (a) and $\frac{l_{k}}{r^{k-1}}$ (b) components for the different values of $k=1,2,3,4,5$.

Figure 6 : Comparison between the theorical model (équation 33) and experimenatl data of Cupressocyparis.

Figure 7 : Several trees growth types: continuous (a), rythmic (b) and alternate (c).

Figure 8: A limetree structure whith Gamma distribution of its apexes. 


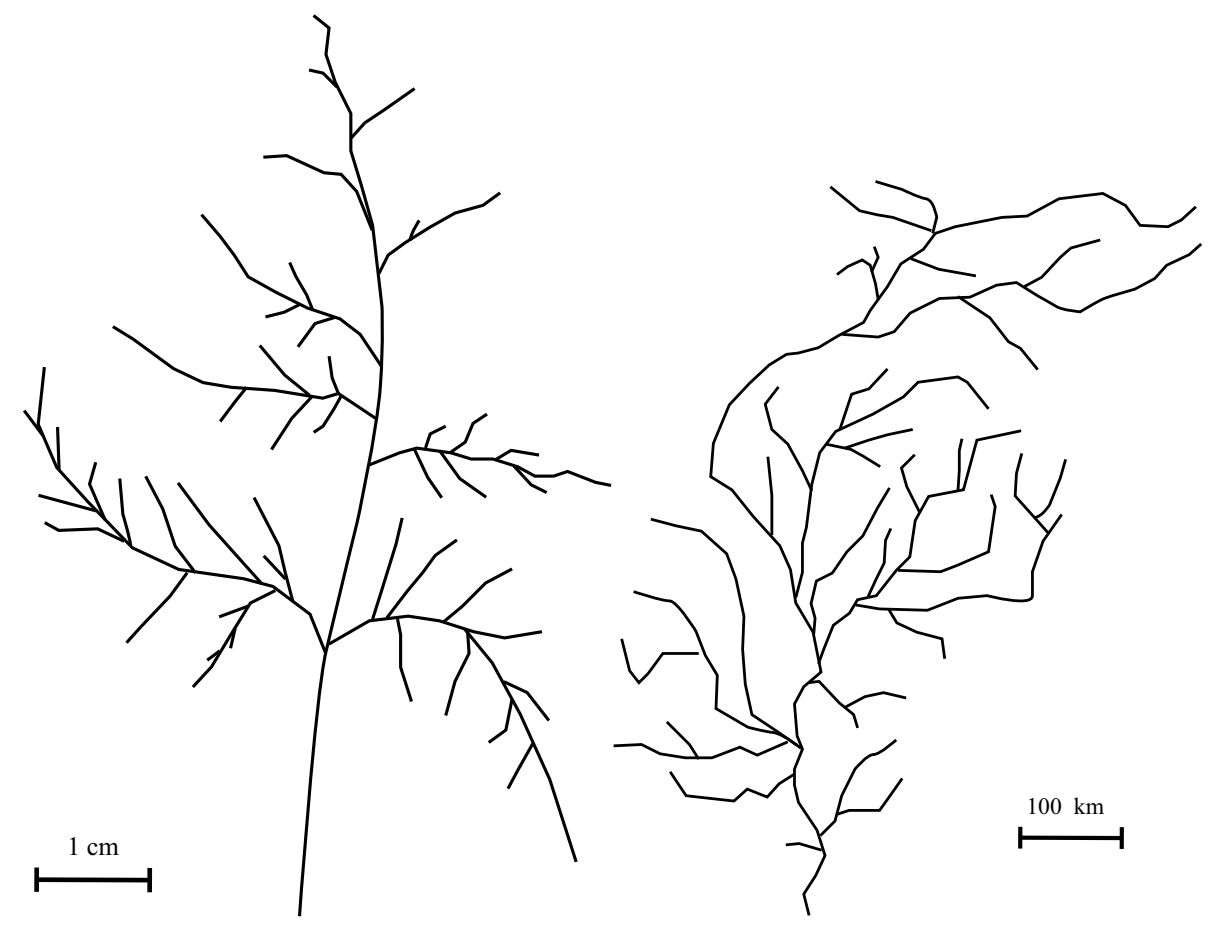

Fig. 1.

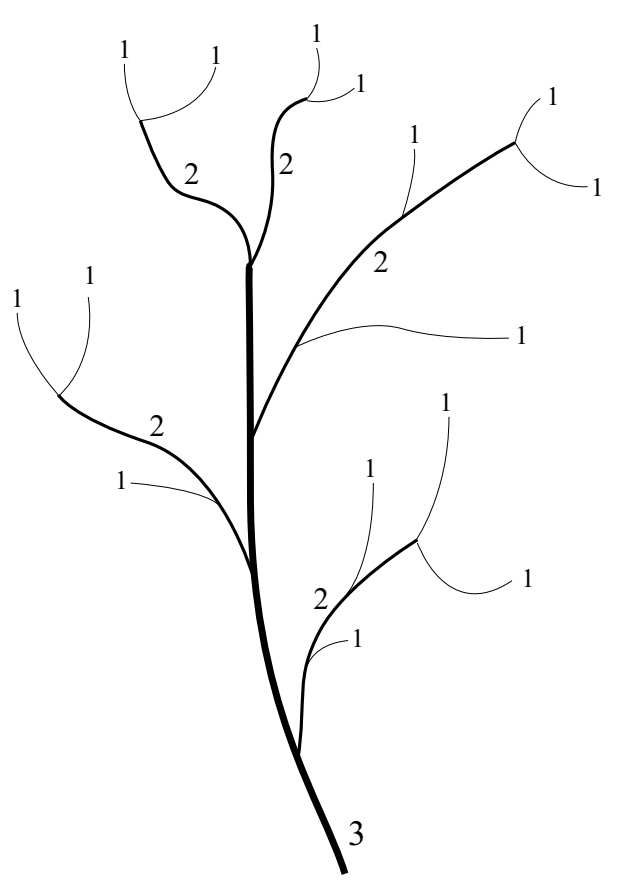

Fig. 2. 


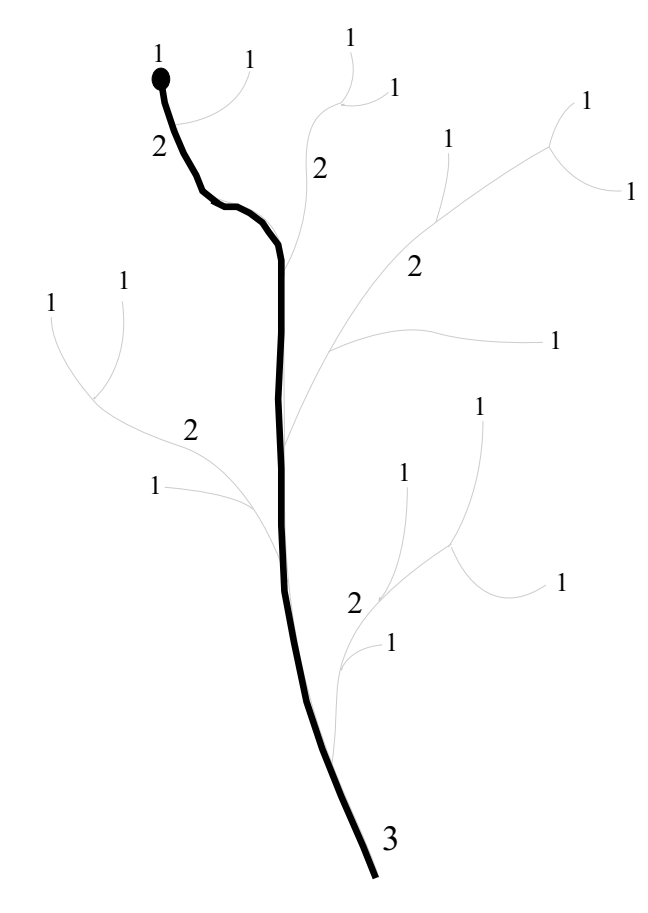

Fig. 3.
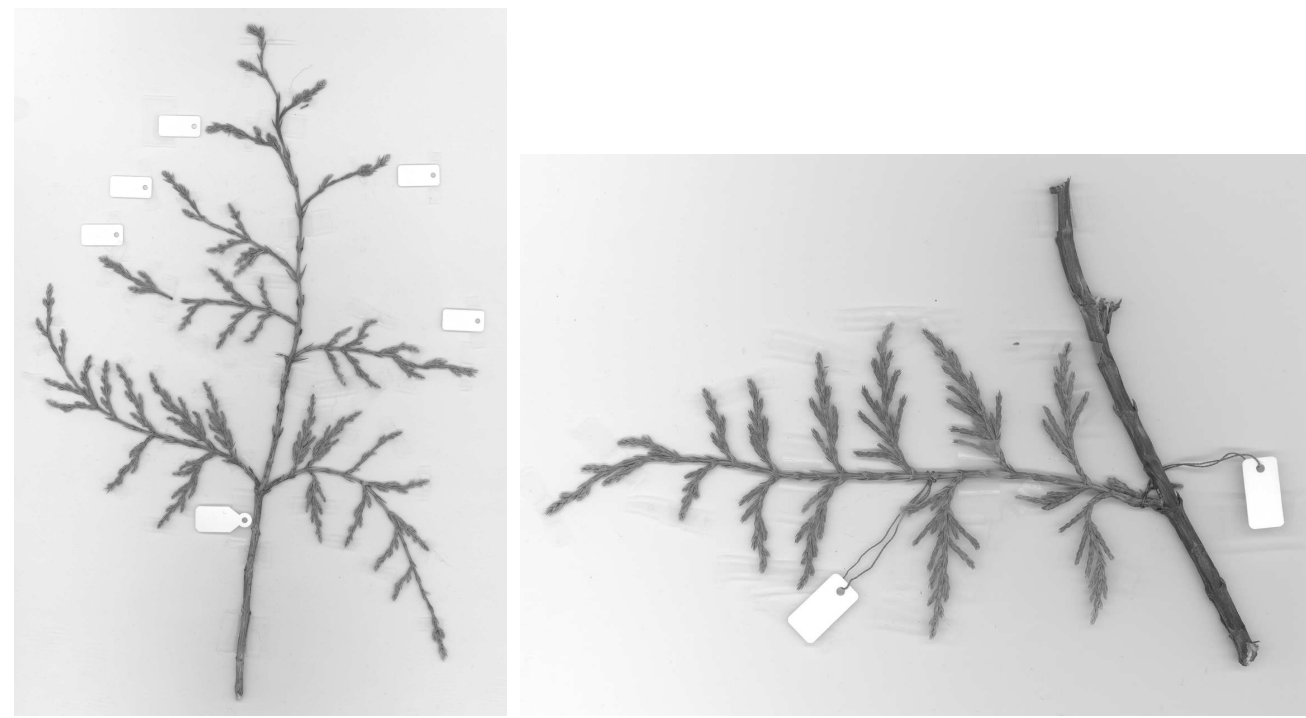

Fig. 4. 


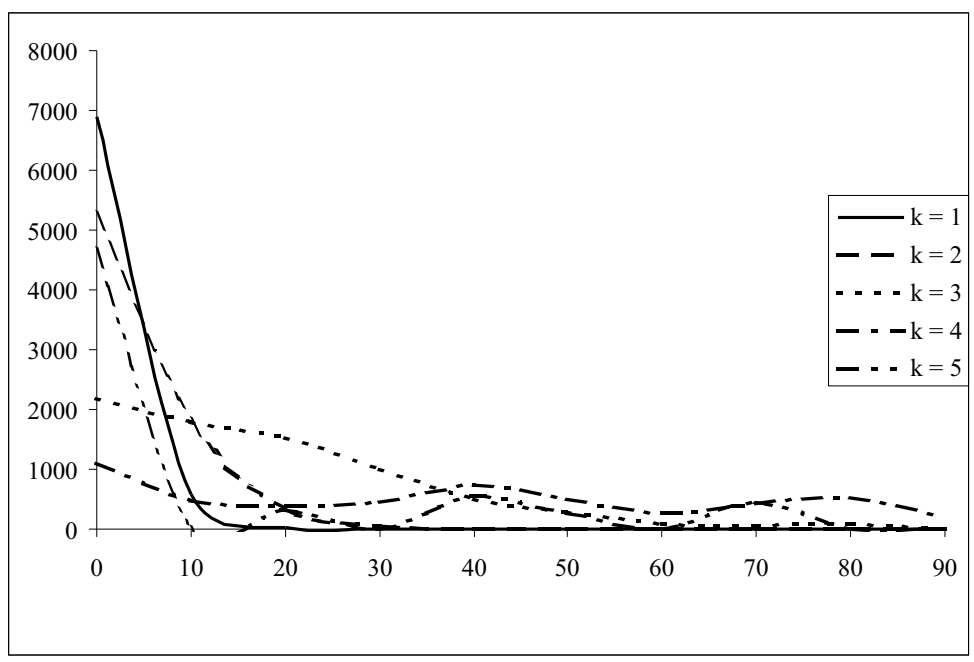

(a)

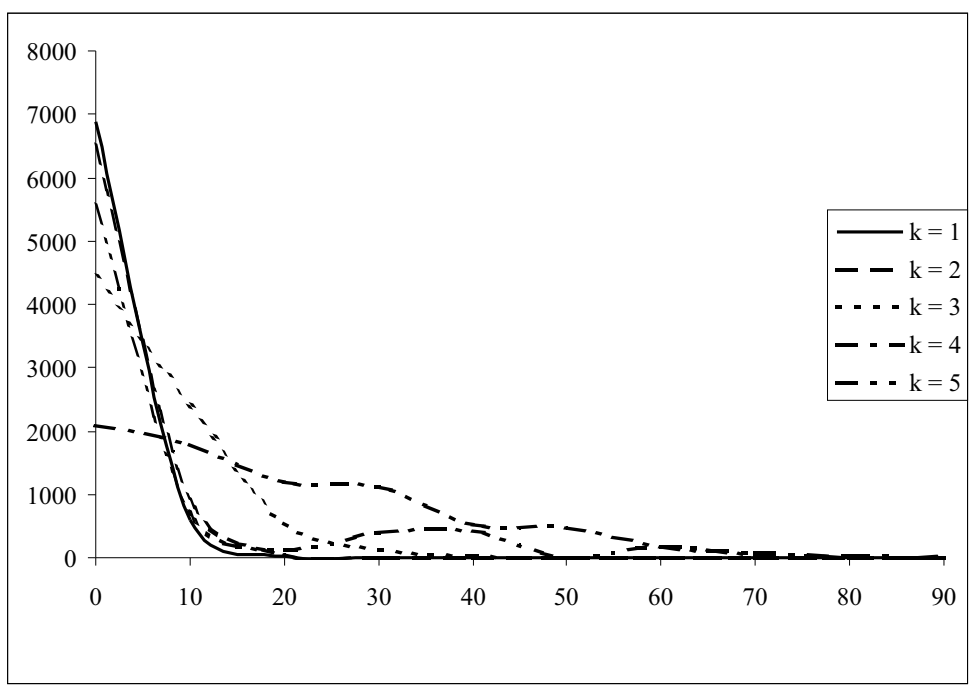

(b)

Fig. 5. 


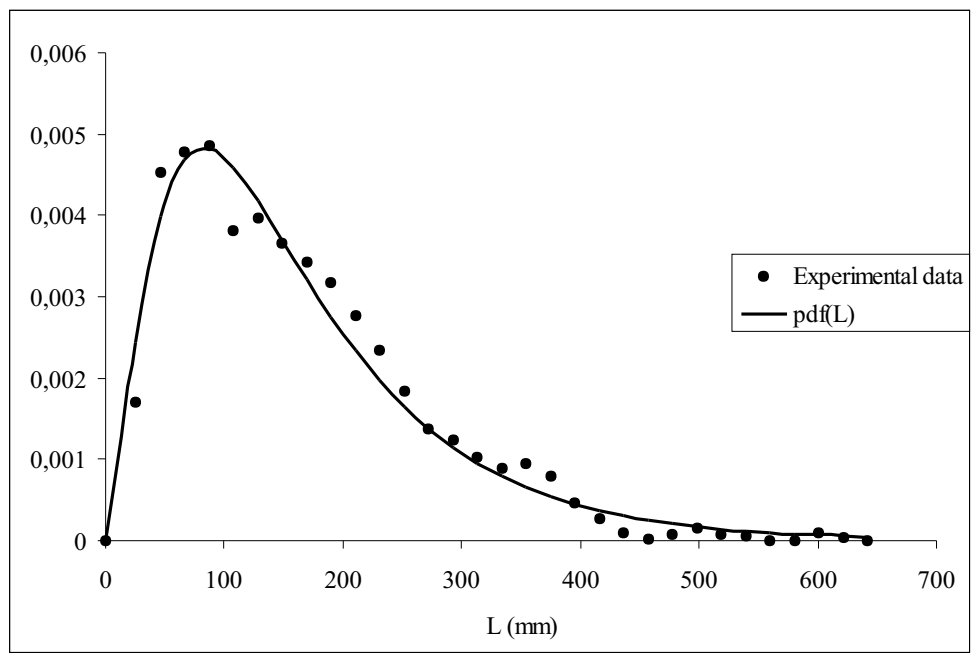

Fig. 6 .

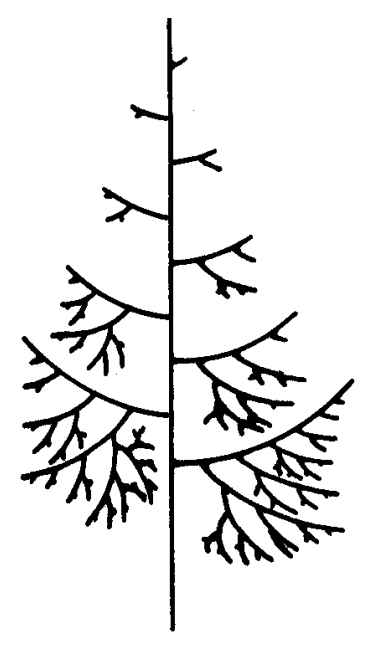

(a)

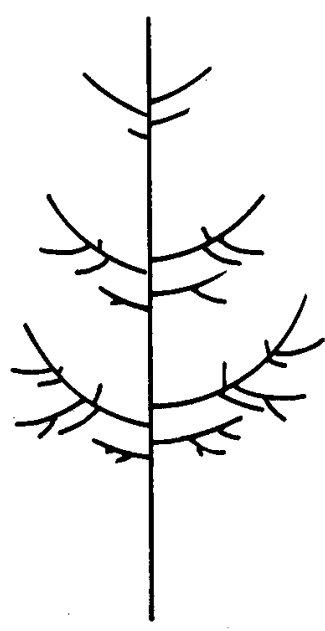

(b)

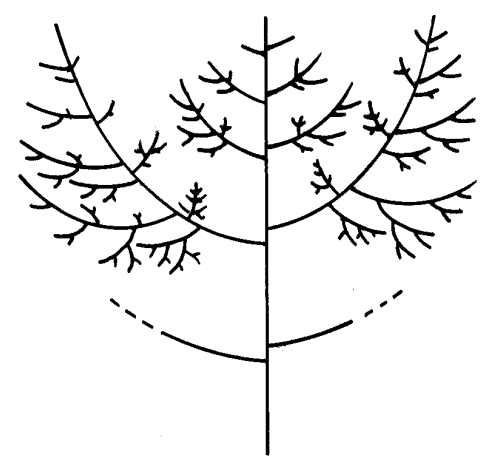

(c)

Fig. 7. 


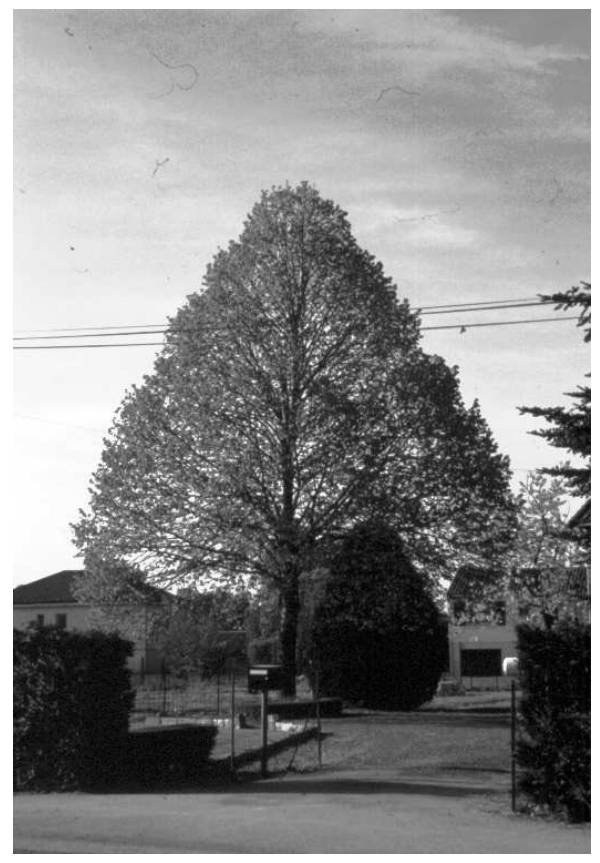

Fig. 8.

\begin{tabular}{|l|l|}
\hline Fractal dimensions & Authors \\
\hline \hline$d=2 \frac{\ln \left(R_{L}\right)}{\ln \left(R_{B}\right)}$ & Feder, 1988 \\
\hline$D=\max \left(1, \frac{\ln \left(R_{L}\right)}{\ln \left(R_{B}\right)}\right)$ & La Barbera et Rosso, 1987, 1989 \\
\hline$D=d \frac{\ln \left(R_{B}\right)}{\ln \left(R_{L}\right)}$ & Tarboton, et al., 1990 \\
\hline$D=\left(\frac{1}{2-d}\right) \frac{\ln \left(R_{B}\right)}{\ln \left(R_{L}\right)}$ & La Barbera et Rosso, 1990 \\
\hline$d=\max \left(1,2 \frac{\ln \left(R_{L}\right)}{\ln \left(R_{A}\right)}\right)$ & Rosso, et al., 1991 \\
\hline$D=\min \left(2,2 \frac{\ln \left(R_{B}\right)}{\ln \left(R_{A}\right)}\right)$ & Rosso, et al., 1991 \\
\hline
\end{tabular}

Table 1

The various findings of the fractal dimension in a stream network. $d$ is a scaling exponent of the stream sinuosity, $D$ is the fractal dimension, $R_{L}, R_{B}$ and $R_{A}$ are respectively the Horton's length ratio, bifurcation ratio and area ratio. 


\begin{tabular}{|l|c|c|c|c|c|}
\hline order $k$ & 1 & 2 & 3 & 4 & 5 \\
\hline \hline$N_{k}$ & 7488 & 1608 & 251 & 20 & 1 \\
\hline$\overline{l_{k}}(\mathrm{~mm})$ & 5.4 & 8.4 & 22.5 & 77.6 & 49.7 \\
\hline$R_{L}$ & 1.56 & 2.66 & 3.44 & 0.64 & \\
\hline$R_{B}$ & 4.65 & 6.4 & 12.55 & 20 & \\
\hline
\end{tabular}

Table 2

Experimental values of the mesured tree parameters (Cupressocyparis). $N_{k}$ is the number of morphometric length of $k$ ordre, $\overline{l_{k}}$ is the average value of the morphometric lenghts of $k$ order, $R_{L}$ and $R_{B}$ are the Horton's ratios. 